\title{
58 Coronaviren
}

\author{
J. Ziebuhr
}

\section{$>$ Einleitung}

Man unterscheidet 5 humanpathogene Coronaviren, die entweder zum Genus Alpha-Coronavirus oder zum Genus Beta-Coronavirus gehören (Subfamilie Coronavirinae, Familie Coronaviridae). Humane Coronaviren (-Tab. 58.1) verursachen akute Erkrankungen des oberen Respirationstraktes, die meist problemlos verlaufen, jedoch gelegentlich zu schweren Pneumonien führen. Eine ursächliche Beteiligung an Gastroenteritiden ist möglich, spielt jedoch klinisch und zahlenmäßig keine große Rolle. Zahlreiche animale Coronaviren sind vor allem an respiratorischen und gastrointestinalen Erkrankungen von Säugetieren und Vögeln beteiligt (•Tab. 58.1).

Der Name der Viren leitet sich vom typischen elektronenmikroskopischen Erscheinungsbild der Virusoberfläche ab, die an eine Krone (lat. »corona«) erinnert. Die Fortsätze dieser Krone werden von den viralen Glykoproteinen, sog. Spikes, gebildet, die in die Virushülle eingelagert sind. Coronaviren sind Plusstrang-RNA-Viren mit den größten Genomen (30 kb) unter allen bekannten RNA-Viren. Sie haben einen Durchmesser von etwa $120 \mathrm{~nm}$. Das helikale Kapsid wird von einer Lipidhülle umgeben, in die mindestens 3 Strukturproteine (SpikeGlykoprotein S, Hüllprotein E, Membranprotein M) eingelagert sind.

\subsection{HCoV-229E, HCoV-OC43, HCoV-NL63, HCoV-HKU1}

Infektionen mit den humanen Coronaviren (HCoV) NL63, 229E, OC43 und HKU1 treten vor allem in den Wintermonaten auf und sind für etwa 5-30\% aller akuten respiratorischen Erkrankungen verantwortlich. Infektionen führen typischerweise $\mathrm{zu}$ Rhinitis, Konjunktivitis, Pharyngitis, gelegentlich auch zu einer Otitis media oder Laryngotracheitis. Die Inkubationszeit beträgt 2-4 Tage, Krankheitssymptome klingen meist nach 1 Woche ab. Eine Mitbeteiligung der unteren Atemwege ist häufiger als noch vor wenigen Jahren angenommen. Stationäre Behandlungen von Patienten mit akuten Infektionen des unteren Respirationstraktes (Pneumonie, Bronchiolitis, Bronchitis) sind bei Kindern in etwa $8 \%$ (bei Erwachsenen $5 \%$ ) der Fälle auf Coronaviren zurückzuführen. Infektionen im Kleinkindalter mit HCoV-NL63 führen häufig auch zu einer Laryngotracheitis (Pseudokrupp). Akute Exazerbationen von Asthma bronchiale infolge Coronavirus-Infektionen sind häufig beschrieben worden.

Typisch ist die zyklische Wiederkehr bestimmter Coronavirus-Stämme im Abstand weniger Jahre. Koinfektionen von

Tab. 58.1 Humane und ausgewählte animale Coronaviren

\begin{tabular}{|c|c|c|c|c|}
\hline $\begin{array}{l}\text { Genus } \\
\text { Virusspezies/-stamm }\end{array}$ & Abkürzung & Wirt & Zellulärer Rezeptor & Krankheiten \\
\hline \multicolumn{5}{|l|}{ Alpha-Coronavirus: } \\
\hline Virus der übertragbaren Gastroenteritis & TGEV & Schwein & Aminopeptidase $\mathrm{N}$ & Gastroenteritis \\
\hline Felines Coronavirus & FCoV, FIPV & Katze & Aminopeptidase $\mathrm{N}$ & Enteritis, Peritonitis \\
\hline Humanes Coronavirus 229E & HCoV-229E & Mensch & Aminopeptidase $\mathrm{N}$ & ARE ${ }^{a}$ \\
\hline Humanes Coronavirus NL63 & HCoV-NL63 & Mensch & $\begin{array}{l}\text { Angiotensin-konvertierendes } \\
\text { Enzym } 2 \text { (ACE2) }\end{array}$ & $\begin{array}{l}\text { ARE, Laryngotracheitis } \\
\text { (Pseudokrupp) }\end{array}$ \\
\hline Virus der porcinen epidemischen Diarrhö & PEDV & Schwein & Aminopeptidase $\mathrm{N}$ & Enteritis \\
\hline \multicolumn{5}{|l|}{ Beta-Coronavirus: } \\
\hline Maus-Hepatitisvirus & MHV & Maus & $\begin{array}{l}\text { mit dem karzinoembryonalen } \\
\text { Antigen (CEA) verwandte Zell- } \\
\text { adhäsionsmoleküle (CEACAM) }\end{array}$ & $\begin{array}{l}\text { Enteritis, ARE, Hepatitis, } \\
\text { Enzephalitis (spezielle Stämme) }\end{array}$ \\
\hline Bovines Coronavirus & $\mathrm{BCoV}$ & Rind & Sialinsäure & Enteritis, ARE \\
\hline Humanes Coronavirus OC43 & HCoV-OC43 & Mensch & Sialinsäure & ARE \\
\hline Humanes Coronavirus HKU1 & HCoV-HKU1 & Mensch & nicht bekannt & ARE \\
\hline SARS-Coronavirus & SARS-CoV & Mensch & $\begin{array}{l}\text { Angiotensin-konvertierendes } \\
\text { Enzym } 2 \text { (ACE2) }\end{array}$ & $\begin{array}{l}\text { schweres akutes Atemwegsyndrom } \\
\text { (SARS) }\end{array}$ \\
\hline \multicolumn{5}{|l|}{ Gamma-Coronavirus: } \\
\hline Virus der infektiösen Bronchitis & IBV & Huhn & Sialinsäure & $\begin{array}{l}\text { Bronchitis, Pneumonie, Nephritis, Infek- } \\
\text { tion des Intestinal- und Genitaltraktes }\end{array}$ \\
\hline
\end{tabular}


Coronaviren mit anderen respiratorischen Viren (v. a. Rhinound Parainfluenzaviren) sind relativ häufig und führen dann zu einem deutlich schwereren Krankheitsbild, nicht selten auch zu einer stationären Behandlung.

Mehr als $80 \%$ aller Erwachsenen besitzen Antikörper gegen humane Coronaviren. Vorausgegangene Infektionen hinterlassen jedoch keine lang anhaltende Immunität, sodass Reinfektionen mit dem gleichen Virus bereits nach 1 Jahr möglich sind. Neugeborene besitzen meist Coronavirus-spezifische Antikörper, die nach 3 Monaten nicht mehr nachweisbar sind. Die Serokonversion erfolgt meist vor Abschluss des 3. Lebensjahres. Es gilt als sicher, dass Coronaviren beim Menschen auch Enteritiden verursachen können. Über deren klinische Bedeutung und die beteiligten Coronavirus-Stämme ist jedoch wenig bekannt.

Für eine gelegentlich diskutierte Rolle humaner Coronaviren bei akuten und chronischen Erkrankungen des ZNS gibt es bisher keinen überzeugenden Beweis, wenngleich RNA von $\mathrm{HCoV}-229 \mathrm{E}$ und HCoV-OC43 in einigen Fällen im ZNS nachgewiesen wurde. Ein überzeugender kausaler Zusammenhang mit einer spezifischen ZNS-Erkrankung wurde bisher nicht hergestellt.

\subsection{SARS-Coronavirus}

Ein zu Beginn des Jahres 2003 noch unbekanntes Coronavirus führte damals zu einer weltweiten Epidemie, die ihren Ausgang in Südchina nahm und sich innerhalb weniger Wochen lokal und international ausbreitete, v. a. in China, Südostasien und Kanada. Weltweit zählte man etwa 9000 Erkrankte und mehr als 800 Tote.

Infektionen mit diesem Virus führten zu einer schweren, häufig beatmungspflichtigen Pneumonie, dem "severe acute respiratory syndrome « (SARS). Durch von der WHO koordinierte, effiziente internationale Zusammenarbeit ließ sich der verantwortliche Erreger innerhalb weniger Wochen identifizieren und seine Verwandtschaft mit bereits bekannten Coronaviren zweifelsfrei beweisen. Er erhielt den Namen SARS-Coronavirus (SARS-CoV).

\section{- Herkunft}

Überzeugenden Hinweisen zufolge haben nahe Verwandte des SARS-CoV ihr natürliches Reservoir in bestimmten Fledermausarten, von denen sie unter möglicher Beteiligung weiterer Zwischenwirte auf den Menschen übertragen wurden. Ein Bindeglied bei der Übertragung von Fledermäusen auf den Menschen war vermutlich der Larvenroller (Paguma larvata), eine Schleichkatzenart, die in China als kulinarische Delikatesse gilt. Bei der Aufzucht dieser Tiere, ihrem Verkauf auf Lebendtiermärkten, beim Schlachten der Tiere und der Essenszubereitung kam es offenbar zur Übertragung des Erregers auf den Menschen. Nach einer weiteren Anpassungsphase in infizierten Personen erreichte ein SARS-CoV-Vorläufervirus dann offenbar zu Beginn des Jahres 2003 eine ausreichend hohe Übertragungsrate. Aufgrund der effizienten Weitergabe von Mensch zu Mensch konnte der Erreger sich in der menschlichen Population etablieren und effizient ausbreiten.
Durch die konsequente Umsetzung hygienischer und gesundheitspolitischer Maßnahmen sowie die schnelle Entwicklung geeigneter diagnostischer Testverfahren zum Nachweis von SARS-CoV ließ sich die weitere Ausbreitung des Erregers bereits nach wenigen Monaten stoppen: Seit 2004 hat es keine neuen Infektionen mehr gegeben. Vermutlich zirkuliert das Virus nicht mehr in der menschlichen Population.

Aktuellen Studien zufolge stellen Fledermäuse ein bedeutsames Reservoir für Coronaviren dar, darunter auch für zahlreiche nahe Verwandte humaner und animaler Coronaviren. Daher wird eine erneute Übertragung SARS-CoV-verwandter oder anderer Coronaviren aus Fledermäusen auf den Menschen oder andere Wirte für möglich gehalten.

\section{Steckbrief}

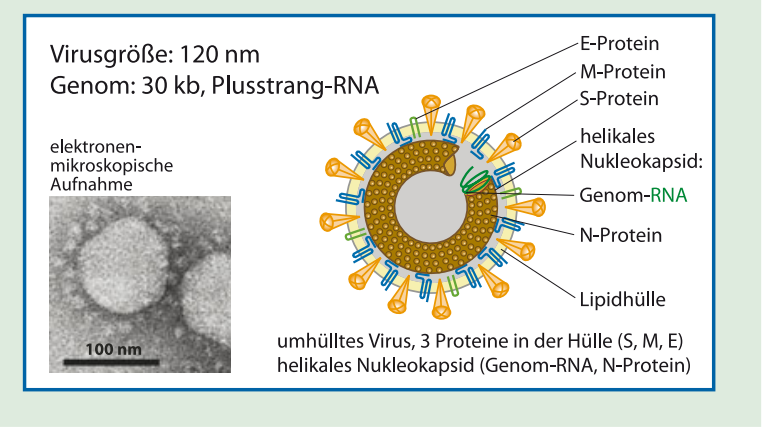

\subsection{Molekularbiologie und Eigenschaften}

\section{- Genomstruktur und -expression}

Coronaviren sind Plusstrang-RNA-Viren und besitzen die größten RNA-Genome (30 kb) aller derzeit bekannten Viren. Neben der Replikation ihres Genoms synthetisieren die Viren (je nach Virusspezies) 4-9 mRNA-Moleküle (- Abb. 58.1), deren 5'- und 3'-Enden mit denen des Genoms identisch sind. Diese "geschachtelten « mRNAs werden auch als »nested set of mRNAs" bezeichnet und haben zur Namensgebung der übergeordneten Virusordnung, Nidovirales (lat. »nidus«: Nest), beigetragen.

Die mRNAs kodieren die Strukturproteine: Spike-Glykoprotein (S), Hüllprotein (E, »envelope«) und Membranprotein (M). Einige Coronaviren (z. B. HCoV-OC43) kodieren eine Hämagglutininesterase (HE) als 4. Strukturprotein sowie eine unterschiedliche Anzahl akzessorischer Proteine, die häufig zur Anpassung an bestimmte Wirte oder zur Pathogenität beitragen.

Zahlreiche Enzyme und Hilfsproteine steuern die coronavirale RNA-Synthese. Sie werden vom Replikasegen kodiert, das aus 2 großen Leserahmen (1a, 1b; • Abb. 58.1) besteht. Die ungewöhnlich komplexe Genexpression erfordert eine ribosomale Leserasterverschiebung zur Expression des 1b-Leserahmens sowie zahlreiche proteolytische Spaltungen der vom Replikasegen kodierten Polyproteine, an denen mehrere Virusproteasen beteiligt sind.

\section{- Stabilität}

Die meisten Coronaviren sind in der Umwelt relativ beständig (bis zu mehreren Tagen). Sie sind empfindlich gegen die im 
Signal für Leseraster-

verschiebung

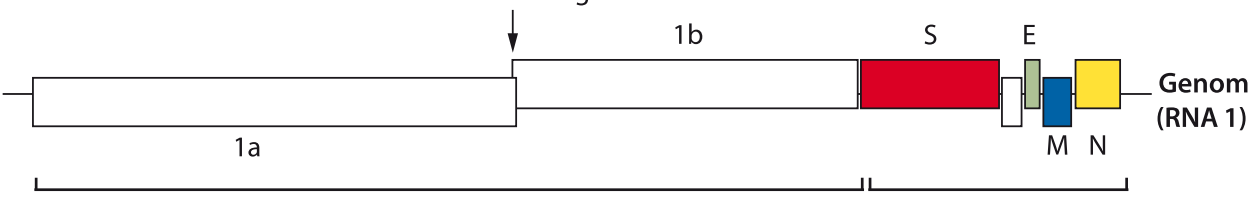

Replikase-Gen

subgenomische mRNAs
Gene für Strukturund akzessorische Proteine
- Abb. 58.1 Coronavirus-Genom und subgenomische RNAs. Die ssRNA (ca. 30 kb) enthält in ihrem 5'-Bereich 2 große Leserahmen (1a u. 1b), die man zusammenfassend als Replikasegen bezeichnet. Dieses kodiert 2 Polyproteine (pp1a u. pp1ab), die virale Proteasen in 16 reife Nichtstrukturproteine spalten (nicht dargestellt). Die Expression des 1b-Leserahmens erfordert eine Verschiebung des ribosomalen Leserasters kurz vor Erreichen des Translations-Stopp-Kodons im 1a-Leserahmen. Die replikasegenkodierten viralen Proteine bilden einen Proteinkomplex mit zahlreichen Untereinheiten, der die virale RNA-Synthese steuert und spezifische Wirtszellfunktionen moduliert. Neben einer RNA-abhängigen RNA-Polymerase enthält dieser Komplex eine Reihe weiterer Enzyme, z. B. Helikase, Primase, Proteasen, Ribonukleasen und Methyltransfera-

Krankenhaus üblichen Desinfektionsmittel, z. B. auf alkoholischer Basis, und lassen sich leicht durch Erhitzen inaktivieren.

\section{- Epidemiologie, Übertragung und Hygienemaßnahmen}

Coronaviren sind für einen großen Teil der akuten respiratorischen Erkrankungen in den Wintermonaten verantwortlich. Sie werden relativ leicht von Mensch zu Mensch durch Tröpfchen und Aerosole übertragen, was nur durch konsequente Hygienemaßnahmen zu verhindern ist. Einige Coronaviren einschließlich SARS-CoV können auch durch Stuhl, Urin und Sekrete ausgeschieden und übertragen werden.

Im Gegensatz zu anderen humanen Coronaviren wurde SARS-CoV in der Regel erst in einem späten Stadium der Erkrankung (SARS) übertragen. Dies erklärt einerseits die besonders hohe Infektionsrate des Klinikpersonals, erleichterte andererseits jedoch auch die Erkennung und Isolierung kontagiöser Patienten und ermöglichte somit die schnelle Beendigung der SARS-Epidemie durch konsequente Anwendung von Hygienemaßnahmen.

\section{- Pathogenese}

Coronaviren dringen über den Nasen-Rachen-Raum ein und vermehren sich dort. Infektionen mit konventionellen Coronaviren (HCoV-229E, HCoV-NL63, HCoV-OC43, HCoV-HKU1) sen. Unterer Bildteil: Virusinfizierte Zellen synthetisieren außerdem subgenomische RNAs, die für die Expression des 3'-Bereichs des Genoms benötigt werden. Da normalerweise in der eukaryotischen Zelle nur der erste, am 5'-Ende einer mRNA gelegene Leserahmen translatiert werden kann, erfordert die Expression der Strukturproteine S, E, M und N (farblich unterlegt) jeweils eine eigene subgenomische RNA. Gleiches gilt für die Translation anderer in diesem Genomabschnitt kodierter Proteine (weiß dargestellt): So wird das S-Protein von der subgenomischen RNA 2 exprimiert (S-Leserahmen ist rot markiert) und die subgenomische RNA 5 für die Expression des M-Proteins benötigt (M-Leserahmen ist blau markiert) und die subgenomische RNA 3 dient zur Expression eines akzessorischen Nichtstrukturproteins

verlaufen in den meisten Fällen als typische »Erkältung «: Husten, Schnupfen, Konjunktivitis, Pharyngitis, gelegentlich auch Laryngotracheitis oder Bronchitis. Es besteht ein allgemeines Krankheitsgefühl mit Müdigkeit, Kopf- und Gliederschmerzen, gelegentlich auch mit Fieber bis $38,5^{\circ} \mathrm{C}$. Infektionen des unteren Respirationstraktes sind möglich, die vor allem bei Koinfektionen mit anderen respiratorischen Erregern zu einer Viruspneumonie führen können.

Infektionen mit SARS-CoV führten zu einem schweren, lebensbedrohlichen Krankheitsbild, das als schweres akutes Atemwegsyndrom (»severe acute respiratory syndrome", SARS) bezeichnet wurde. Typische Symptome waren plötzliches Fieber, Myalgien, trockener Husten, schweres Krankheitsgefühl und Schüttelfrost, jedoch kein Schnupfen oder Halsschmerzen. Im weiteren Verlauf der Erkrankung kam es zu Atemnot wegen mangelhafter Sauerstoffsättigung im Blut, häufig auch zu Durchfall. Während der Epidemie des Jahres 2003 mussten 20-30 \% der Patienten intensivmedizinisch behandelt werden und etwa $10 \%$ der Infizierten verstarben. Das Virus infiziert in erster Linie die Zellen des Alveolarepithels, insbesondere Typ-1-Pneumozyten, war jedoch häufig auch in anderen Organen wie Niere, Leber und Dünndarm nachweisbar.

Die Ursachen der massiven Lungenschädigung sind nicht ganz klar. Es gibt jedoch deutliche Hinweise auf eine Beteiligung 
immunpathologischer Mechanismen. Die SARS-CoV-Infektion von Makrophagen, konventionellen dendritischen Zellen und vielen anderen Zellen führt nicht zur Induktion einer Typ-I-Interferon-Antwort. Daher vermutet man, dass ein Versagen der Sofortantwort des Immunsystems den beobachteten starken Anstieg der Virusreplikation während der ersten 10 Tage erlaubte, bevor dann die adaptiven Immunmechanismen voll aktiviert wurden und die Virusreplikation in den meisten Fällen unter Kontrolle brachten. Zu diesem späten Zeitpunkt war bereits ein massiver Einstrom von Makrophagen und neutrophilen Granulozyten erfolgt, begleitet von einer starken Erhöhung von Zytound Chemokinen.

Das histopathologische Bild zeigte eine diffuse alveoläre Schädigung, die Ablösung von Pneumozyten, massive inflammatorische Infiltrationen, Ödeme und die Bildung hyaliner Membranen. Diese massive Schädigung der Lungenalveolen gilt als Hauptursache der hohen Letalität.

\section{In Kürze}

\section{Coronaviren}

Molekularbiologie 5 humane Coronaviren sind bekannt, die entweder zum Genus Alpha-Coronavirus (HCoV-229E, HCoV-NL63) oder zum Genus Beta-Coronavirus (HCoV-OC43, HCoV-HKU1, SARS-CoV) gehören. ss(+)-RNA-Virus mit helikalem Kapsid. Hülle aus mindestens 3 Proteinen, von denen das SpikeProtein zur typischen Struktur (»Corona«) im Elektronenmikroskop beiträgt. Größtes bekanntes RNA-Genom. Ungewöhnlich komplexe Replikationsstrategie mit speziellen RNA-Synthesemechanismen und zahlreichen Enzymen, die zum überwiegenden Teil bei anderen Viren nicht vorhanden sind. Vorkommen Coronaviren sind typische Erreger oberer Atemwegerkrankungen. SARS-CoV wurde 2002/2003 auf den Menschen übertragen (ursprünglich von Fledermäusen, wahrscheinlich auf Umweg über bestimmte Schleichkatzen), danach durch Reisende in viele Länder verbreitet. Es zirkuliert nicht mehr in der menschlichen Population. Es gibt jedoch bedeutsame tierische Reservoire verschiedenster Coronavirus-Arten der Gat-

\subsection{Diagnostik und Therapie}

Antikörper gegen Coronaviren lassen sich in verschiedenen Verfahren nachweisen, sind jedoch meist von geringer Aussagekraft. Die Diagnose erfolgt mittels RT-PCR, z. B. aus Nasen-RachenAbstrichen, BAL oder Stuhl. PCR-basierte Multiplextestverfahren zum Nachweis humaner Coronaviren und anderer respiratorischer Viren finden zunehmend Anwendung. Die Anzucht der meisten humanen Coronaviren ist schwierig und wird deshalb nicht routinemäßig durchgeführt. SARS-CoV ist eine Ausnahme: Es lässt sich problemlos auf Vero-Zellen vermehren.

DD: Influenza, RSV-Bronchiolitis, Pneumonien anderer Ätiologie, u. a. Metapneumovirus, Paramyxoviren, Rhinoviren, Chlamydia pneumoniae.

Die Therapie erfolgt symptomatisch bzw. beschränkt sich auf die Behandlung möglicher bakterieller Superinfektionen. Therapieversuche bei SARS mit Ribavirin und Typ-I-Interferonen wurden unternommen, eine therapeutische Wirksamkeit war jedoch nicht überzeugend nachweisbar.

tungen Alpha- und Beta-Coronavirus in Fledermäusen (weltweit) sodass ein erneutes Überspringen von Coronaviren auf den Menschen nicht auszuschließen ist.

Übertragung Durch Tröpfchen und Aerosole. Bei SARS-CoV und einigen anderen Coronaviren auch durch Sekrete und Stuhl.

Pathogenese und Klinik Eindringen über den Nasen-Rachen- in den Bronchialraum. Bei den 4 verbreiteten humanen Coronaviren: Erkrankungen der oberen Luftwege (Schnupfen, Pharyngitis, Laryngotracheitis, Bronchitis), gelegentlich auch Pneumonien. Bei SARS: schwere Pneumonie, hämatogene Streuung, virus- und immunsystembedingter Zellschaden.

Diagnose RT-PCR. Antikörpernachweis zwar möglich, aber meist nicht aussagekräftig, da Serokonversion bereits im frühen Kindesalter erfolgt, die jedoch keinen wirksamen Schutz vor Reinfektionen bietet.

Prävention Bei SARS und anderen schweren Coronavirus-Infektionen: Isolierung der Kranken und Kontaktpersonen, strikte Hygienemaßnahmen. 